\title{
HEXAGONAL VERSUS ORTHOGONAL LATTICES: A NEW COMPARISON USING APPROXIMATION THEORY
}

\section{Laurent Condat*}

\author{
Laboratoire LIS - Fédération ELESA \\ Av. Félix Viallet \\ 38031 Grenoble cedex, France \\ E-mail: laurent.condat@lis.inpg.fr
}

\begin{abstract}
We provide a new comparison between hexagonal and orthogonal lattices, based on approximation theory. For each of the lattices, we select the "natural" spline basis function as generator for a shift-invariant function space; i.e., the tensor-product B-splines for the orthogonal lattice and the non-separable hex-splines for the hexagonal lattice. For a given order of approximation, we compare the asymptotic constants of the error kernels, which give a very good indication of the approximation quality. We find that the approximation quality on the hexagonal lattice is consistently better, when choosing lattices with the same sampling density. The area sampling gain related to these asymptotic constants quickly converges when the order of approximation of the basis functions increases. Surprisingly, nearestneighbor interpolation does not allow to profit from the hexagonal grid. For practical purposes, the second-order hex-spline (i.e., constituted by linear patches) appears as a particularly useful candidate to exploit the advantages of hexagonal lattices when representing images on them.
\end{abstract}

\section{INTRODUCTION}

Digital image processing systems require a sampling strategy to represent and manipulate two-dimensional (2-D) data. The orthogonal lattice is commonly used for this purpose. Hexagonal sampling, although less popular, offers many theoretical advantages for image processing tasks: the twelve-fold symmetry for more isotropic treatment, and the six-connectivity allows Jordan's theorem to be satisfied with applications in edge detection and pattern recognition [1].

An important issue in image processing is the link between the discrete and the continuous domain: operations such as resampling ask for a continuous model to be fitted on the discrete samples; e.g., for image resizing, rotating, or other geometric operations. Spline models have shown to be particularly suitable for this purpose $[2,3]$. This is

\footnotetext{
* This work was initiated during the first author's visit at BIG, EPFL supported by Région Rhône-Alpes (EURODOC grant).
}

\author{
Dimitri Van De Ville, Thierry Blu
}

\author{
Biomedical Imaging Group \\ École Polytechnique Fédérale de Lausanne (EPFL) \\ CH-1015 Lausanne EPFL, Switzerland
}

due to their tunability and optimal approximation capabilities $[4,5]$.

Recently, a novel spline family, especially designed for hexagonal lattices, has been proposed [6]. These splines, named "hex-splines", arise naturally from the shape of the Voronoi lattice cell. In the hexagonal case, they preserve the twelve-fold symmetry since they are built from the hexagonal tiling cell by successive convolutions. The hex-spline family includes the classical tensor-product B-splines as a special case. Hex-splines were already successfully applied to some printing applications [7].

In this paper, we compare hexagonal and orthogonal lattices with the same sampling density. For each of them, we deploy the "natural" spline basis function; i.e., the tensorproduct B-splines for the orthogonal lattice and the hexsplines for the hexagonal lattice. For a given order of approximation of the basis functions, the asymptotic constants of the error kernel are useful indicators to compare the approximation quality. We show that the hexagonal lattice is consistently better, in particular starting from second-order splines. This result confirms the classical observation that isotropic band-limited images can be represented more efficiently on a hexagonal lattice than on an orthogonal lattice. However, our new comparison does not require the bandlimited assumption of the signal, neither the use of ideal (sinc-like) basis functions.

\section{2-D SPLINES ON PERIODIC LATTICES}

Some conventions are used throughout this paper. Vectors are denoted as $\mathbf{x}=\left[\begin{array}{ll}x_{1} & x_{2}\end{array}\right]^{\mathrm{T}} \in \mathbb{R}^{2}$. The 2-D Fourier transform of a function $f(\mathbf{x})$ is defined as $\hat{f}(\boldsymbol{\omega})=\int_{\mathbb{R}^{2}} f(\mathbf{x})$ $\exp \left(-j \boldsymbol{\omega}^{\mathrm{T}} \mathbf{x}\right) \mathrm{d} \mathbf{x}$.

A 2-D periodic lattice $\Lambda$ is characterized by two independant vectors $\mathbf{r}_{1}$ and $\mathbf{r}_{2}$ grouped in a matrix $\mathbf{R}=\left[\begin{array}{ll}\mathbf{r}_{1} & \mathbf{r}_{2}\end{array}\right]$, so the lattice sites are given by $\mathbf{R k} ; \mathbf{k} \in \mathbb{Z}^{2}$. We define $\Omega=|\operatorname{det} \mathbf{R}|$. Then the density of the lattice is $1 / \Omega$ [lattice sites per unit surface]. We also define $\hat{\Lambda}$, the dual lattice of $\Lambda$, whose matrix is $\hat{\mathbf{R}}=\left(\mathbf{R}^{-1}\right)^{\mathrm{T}}$. The effect of sampling a function $f(\mathbf{x})$ on $\Lambda$ is to replicate its spectrum $\hat{f}(\boldsymbol{\omega})$ on the 
lattice sites $2 \pi \hat{\mathbf{R}} \mathbf{k}$.

A discrete sequence $h=(h[\mathbf{k}])_{\mathbf{k} \in \mathbb{Z}^{2}}$ defined on a lattice $\Lambda$ can be regarded as a Dirac comb $\sum_{\mathbf{k} \in \mathbb{Z}^{2}} h[\mathbf{k}] \delta(\mathbf{x}-\mathbf{R k})$, where $\delta(\mathbf{x})$ is the Dirac distribution located at the origin $\mathbf{0 .}$ Therefore its periodized Fourier transform $\hat{h}(\boldsymbol{\omega})$ is defined as $\hat{h}(\boldsymbol{\omega})=\sum_{k \in \mathbb{Z}^{2}} h[\mathbf{k}] \exp \left(-j \boldsymbol{\omega}^{\mathrm{T}} \mathbf{R} \mathbf{k}\right)$.

A well-defined tiling cell of a lattice is the Voronoi cell, consisting of all points closer to $\mathbf{0}$ than to any other lattice site. The Voronoi cell of a lattice characterized by $\mathbf{R}$ has surface area $\Omega$ and can be represented by the indicator function $\chi_{\mathbf{R}}(\mathbf{x})$ :

$$
\chi_{\mathbf{R}}(\mathbf{x})= \begin{cases}1, & \mathbf{x} \in \text { Voronoi cell, } \\ 1 / m_{\mathbf{x}}, & \mathbf{x} \in \text { edge of the Voronoi cell, } \\ 0, & \mathbf{x} \notin \text { Voronoi cell }\end{cases}
$$

where $m_{\mathbf{x}}$ is the number of lattice points to which $\mathbf{x}$ is equidistant. Note that $\chi_{\mathbf{R}}(\mathbf{x})$ tiles the plane by definition.

Splines suitable for the regular orthogonal lattice, described by the unity matrix $\mathbf{R}=\mathbf{I}_{2}$, can easily be obtained by using the tensor-product of two one-dimensional B-splines $\beta^{n}(\mathbf{x})=\beta^{n}\left(x_{1}\right) \beta^{n}\left(x_{2}\right)$. The superscript $n$ refers to the $n$-th degree of piecewise polynomials or to the $n+1$ th order of approximation [4]. We recall that the 1-D Bsplines $\beta^{n}(x)$ are constructed by successive convolutions: $\beta^{n}(x)=\beta^{n-1}(x) * \beta^{0}(x)$ where

$$
\beta^{0}(x)= \begin{cases}1, & x \in\left(-\frac{1}{2}, \frac{1}{2}\right) \\ 1 / 2, & |x|=\frac{1}{2} \\ 0 & \text { else. }\end{cases}
$$

$\beta^{0}(x)$ can be considered as the indicator function of the "Voronoi interval" for the 1-D lattice $\mathbb{Z}$. Therefore it is natural to extend the construction to any 2-D lattice, by defining the first-order hex-spline from the Voronoi cell as $\eta_{1}(\mathbf{x})=\chi_{\mathbf{R}}(\mathbf{x})$. Note that this spline is normalized to the surface area of the Voronoi cell: $\int_{\mathbb{R}^{2}} \eta_{1}(\mathbf{x}) \mathrm{d} \mathbf{x}=\Omega$. Hexsplines of higher orders are constructed by successive convolutions

$$
\eta_{L+1}(\mathbf{x})=\frac{\eta_{1} * \eta_{L}(\mathbf{x})}{\Omega}, \quad L \geq 1 .
$$

When the orthogonal lattice is considered, the hex-spline $\eta_{L}(\mathbf{x})$ simply reverts to the separable B-spline $\beta^{L-1}(\mathbf{x})$.

Consider now the uniform hexagonal lattice (of the second type) $\Lambda_{\text {hex }}$ and the orthogonal lattice $\Lambda_{\text {orth }}$, both with density 1 , and described by the matrices:

$$
\mathbf{R}_{\text {hex }}=\sqrt{\frac{2}{\sqrt{3}}}\left[\begin{array}{cc}
\sqrt{3} / 2 & 0 \\
1 / 2 & 1
\end{array}\right], \quad \mathbf{R}_{\text {orth }}=\left[\begin{array}{ll}
1 & 0 \\
0 & 1
\end{array}\right] .
$$

These two lattices and their Voronoi cells are depicted in Fig. 1. The hex-spline of second order, constructed on $\Lambda_{\text {hex }}$, is shown in Fig. 2. As $L$ increases, $\eta_{L}$ becomes more and more Gaussian-like. For more interesting properties of the hex-splines (analytical form, Riesz basis, convexity, partition of unity, ...), we refer to [6].
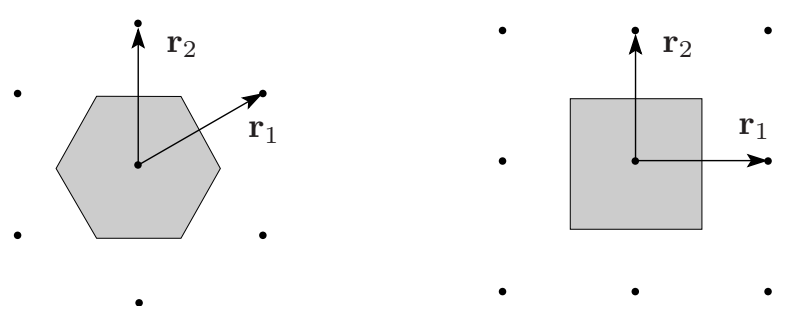

Fig. 1. The hexagonal $\Lambda_{\text {hex }}$ and orthogonal $\Lambda_{\text {orth }}$ lattices, and their associated Voronoi cells.

\section{APPROXIMATION IN 2-D SHIFT-INVARIANT FUNCTION SPACES}

A linear shift-invariant function space $V_{\mathbf{R}}(\varphi)$ (e.g., a spline space), spanned by shifted versions of a generator $\varphi(\mathbf{x})$, contains all the signals

$$
s(\mathbf{x})=\sum_{\mathbf{k} \in \mathbb{Z}^{2}} c[\mathbf{k}] \varphi(\mathbf{x}-\mathbf{R} \mathbf{k}) ; \quad(c[\mathbf{k}])_{\mathbf{k} \in \mathbb{Z}^{2}} \in \ell_{2}\left(\mathbb{Z}^{2}\right) .
$$

When approximating an original function $f \in L_{2}\left(\mathbb{R}^{2}\right)$ by a representative $s \in V_{\mathbf{R}}(\varphi)$, the coefficients $c[\mathbf{k}]$ are determined using a prefilter $\tilde{\varphi}$ as

$$
c[\mathbf{k}]=\int_{\mathbb{R}^{2}} f(\mathbf{x}) \tilde{\varphi}(\mathbf{x}-\mathbf{R} \mathbf{k}) \mathrm{d} \mathbf{x} .
$$

The optimal choice for the prefilter, minimizing the $L_{2}$ distance $\|f-s\|_{L_{2}} \triangleq \int_{\mathbb{R}^{2}}|f(\mathbf{x})-s(\mathbf{x})|^{2} \mathrm{~d} \mathbf{x}$ corresponds to the orthogonal projection of $f$ onto $V_{\mathbf{R}}(\varphi)$, thereafter denoted $\mathcal{P}_{\mathbf{R}} f$. The associated prefilter of this choice is $\tilde{\varphi}=\varphi_{d}$, where $\varphi_{d}$ is called the dual function of $\varphi$, defined in the Fourier domain by

$$
\hat{\varphi}_{d}(\boldsymbol{\omega})=\frac{\hat{\varphi}(\boldsymbol{\omega})}{\hat{a}_{\varphi}(\boldsymbol{\omega})},
$$

where $\hat{a}_{\varphi}(\boldsymbol{\omega})$ is the Fourier transform of the autocorrelation

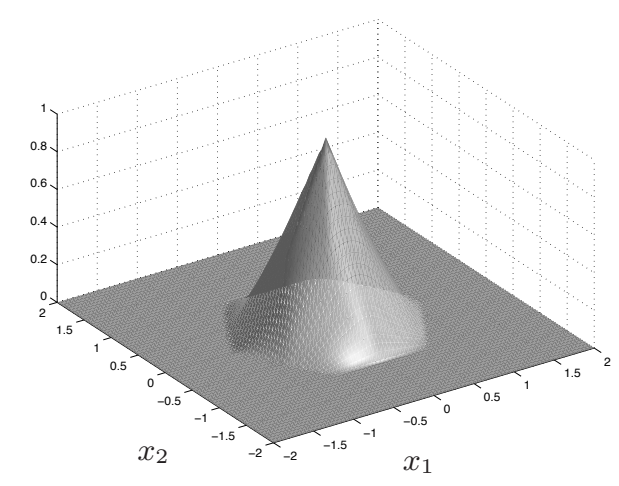

Fig. 2. Hex-spline $\eta_{2}(\mathbf{x})$ for the lattice $\Lambda_{\text {hex }}$. 
sequence $\left(a_{\varphi}[\mathbf{k}]\right)_{\mathbf{k} \in \mathbf{Z}^{2}}$ of $\varphi$ defined by

$$
a_{\varphi}[\mathbf{k}]=\int_{\mathbb{R}^{2}} \varphi(\mathbf{x}) \varphi(\mathbf{x}+\mathbf{R} \mathbf{k}) \mathrm{d} \mathbf{x} .
$$

In this paper, we want to characterize the approximation error $\left\|\mathcal{P}_{\mathbf{R}} f-f\right\|_{L_{2}}$. Approximation theory provides us with a convenient way to quantify this error. A remarkable result from [5] is that the approximation error can be predicted very accurately by the quantity

$$
\varepsilon_{f}=\frac{1}{2 \pi} \sqrt{\int_{\mathbb{R}^{2}}|\hat{f}(\boldsymbol{\omega})|^{2} E(\boldsymbol{\omega}) \mathrm{d} \boldsymbol{\omega}}
$$

where the error kernel $E(\boldsymbol{\omega})$ can be written as

$$
E(\boldsymbol{\omega})=1-\frac{|\hat{\varphi}(\boldsymbol{\omega})|^{2}}{\hat{a}_{\varphi}(\boldsymbol{\omega})} .
$$

As in practice most images are essentially lowpass, the approximation error is controlled by the behavior of $E(\omega)$ around $\boldsymbol{\omega}=\mathbf{0}$. It has been shown in [5] that $E(\boldsymbol{\omega}) \propto \boldsymbol{\omega}^{2 L}$ if and only if the well-known Strang-Fix conditions of order $L$ [8] are satisfied:

$\hat{\varphi}(\mathbf{0}) \neq 0$ and $\hat{\varphi}^{(n)}(2 \pi \hat{\mathbf{R}} \mathbf{k})=0$ for $\left\{\begin{array}{l}\mathbf{k} \neq \mathbf{0} \\ n=0 \ldots L-1\end{array}\right.$

in which case $\varphi$ is said to have approximation order $L$.

The Strang-Fix conditions also imply that, if the sampling lattice is made finer by a scaling factor $\lambda \in \mathbb{R}^{+}$(corresponding to the lattice matrix $\lambda \mathbf{R}$ ), and $\lambda$ tends to zero, then the approximation error has an $L$ th-order decay; i.e. $\left\|\mathcal{P}_{\lambda \mathbf{R}} f-f\right\|_{L_{2}} \propto \lambda^{L}$. Thus the asymptotic behavior tells us how well the approximation converges to the original function $f$ when the sampling lattice is made finer.

In order to compare approximation schemes with the same approximation order, we have to go one step further and evaluate the asymptotic constant $C(\theta)$ so that

$$
E(\boldsymbol{\omega})=C(\theta)\|\boldsymbol{\omega}\|^{2 L} \quad \text { as } \boldsymbol{\omega} \rightarrow \mathbf{0},
$$

where $\theta$ is defined by the coordinates changes $\left(\omega_{1}, \omega_{2}\right)=$ $(\|\boldsymbol{\omega}\| \cos (\theta),\|\boldsymbol{\omega}\| \sin (\theta)) . C(\theta)$ depends on both $\varphi$ and $\mathbf{R}$.

In the next section, we compute the asymptotic constants for the hex-splines on the hexagonal lattice. Then we compare them with the constants for the separable splines on the orthogonal lattice, so as to show that the hexagonal lattice offers a better approximation quality.

\section{HEXAGONAL VERSUS ORTHOGONAL: APPROXIMATION QUALITY}

Let us first consider the orthogonal lattice $\Lambda_{\text {orth }}$ and the $L$-th order tensor-product B-spline $\beta^{L-1}(\mathbf{x})$. The corresponding asymptotic constant of (12), denoted $C_{L}^{\text {orth }}$, does not depend on $\theta$. Using the Riemann-Zeta function $\zeta(x)=\sum_{n=1}^{\infty} \frac{1}{n^{x}}$, we have [5]

$$
C_{L}^{\mathrm{orth}}=\frac{2 \zeta(2 L)}{(2 \pi)^{2 L}} .
$$

Here, we are particularly interested in the approximation quality of the hex-splines $\eta_{L}$ for the lattice matrix $\mathbf{R}_{\text {hex }}$. We have obtained the corresponding constant $C_{L}^{\text {hex }}(\theta)$ (proof in Appendix) as

$$
\begin{aligned}
& C_{L}^{\mathrm{hex}}(\theta)= \\
& \frac{6}{\left(72 \sqrt{3} \pi^{2}\right)^{L}} \zeta(2 L) \sum_{n=-\lfloor L / 3\rfloor}^{\lfloor L / 3\rfloor}\left(\begin{array}{l}
2 L \\
3 n+L
\end{array}\right)(-1)^{n} \cos (6 n \theta) \\
& +\left(\frac{27}{8 \sqrt{3} \pi^{4}}\right)^{L} \sum_{\substack{\mathbf{k} \in \mathbb{Z}^{2} \\
k_{1}+k_{2} \neq \equiv 0[3]}}\left[\frac{\left(\frac{\sin (\theta+2 \pi / 3)}{k_{1}+k_{2}}-\frac{\sin (\theta-2 \pi / 3)}{k_{1}-2 k_{2}}\right)}{2 k_{1}-k_{2}}\right]^{2 L},
\end{aligned}
$$

where $0[3]$ stands for all multiples of $3 . C_{L}^{\text {hex }}(\theta)$ is $\frac{\pi}{3}$ periodic, and its maximum is attained at $\theta=0$. So we define $C_{L}^{\text {hex* }} \triangleq C_{L}^{\text {hex }}(0)$. This new constant can be interpreted as the worst case behavior and has the following closed form (again see Appendix):

$$
C_{L}^{\mathrm{hex} *}=\frac{2 \zeta(2 L)^{2}\left(9^{L}-1\right)^{2}}{\left(32 \sqrt{3} \pi^{4}\right)^{L}}+\frac{4 \zeta(2 L)}{\left(24 \sqrt{3} \pi^{2}\right)^{L}} .
$$

When $L \rightarrow \infty$, we have the asymptotic equivalence $C_{L}^{\mathrm{hex*}} \sim\left(\frac{27 \sqrt{3}}{32 \pi^{4}}\right)^{L}$, which is a good approximation of (15): for $L=4$, the value is exact within $0.9 \%$.

To compare the asymptotic constants (13) and (15), one typically considers the "area sampling gain" $\lambda_{L}^{2}$, defined as the change in sampling density to make the two constants equal. Specifically, scaling $\mathbf{R}$ to $\lambda_{L} \mathbf{R}$ changes the corresponding density $1 / \Omega$ to $1 /\left(\lambda_{L}^{2} \Omega\right)$. Consequently, the asymptotic constant $C(\theta)$ becomes $\lambda_{L}^{2 L} C(\theta)$. The area sampling gain of the hexagonal over the orthogonal lattice must make

$$
\lambda_{L}^{2 L} C_{L}^{\text {hex* }}=C_{L}^{\text {orth }},
$$

from which we find $\lambda_{L}^{2}=\sqrt[L]{\frac{C_{L}^{\text {orth }}}{C_{L}^{\text {hex* }}}}$. The values for the first four orders are listed in Tab. 1. When $L$ tends to $\infty, \lambda_{L}^{2} \rightarrow$ $\frac{8 \pi^{2}}{27 \sqrt{3}}$, and this value is already achieved for $L=4$ within $0.1 \%$.

\section{DISCUSSION}

The area sampling gain suggests that we could reduce the number of samples on the hexagonal lattice with about $40 \%$, and still obtain the same asymptotic constant as for the orthogonal lattice. Although spectacular at first sight, this result should be interpreted with caution. The asymptotic constant only gives the trend of the error kernel around the 
Table 1. Area sampling gain of the hexagonal lattice versus the orthogonal lattice, for a given approximation order $L$.

\begin{tabular}{ccc}
\hline $\begin{array}{c}\text { approximation } \\
\text { order } L\end{array}$ & $\begin{array}{c}\text { area sampling } \\
\text { gain } \lambda_{L}^{2}\end{array}$ & $\begin{array}{c}\text { improvement of } \Lambda_{\text {hex }} \\
\text { over } \Lambda_{\text {ort }}(\%)\end{array}$ \\
\hline 1 & $\frac{3 \sqrt{3}}{5} \approx 1.039$ & 3.8 \\
2 & $\frac{3 \sqrt{2}}{\sqrt{7}} \approx 1.604$ & 37.7 \\
3 & $\approx 1.676$ & 40.3 \\
4 & $\approx 1.686$ & 40.7 \\
$\infty$ & $\frac{8 \pi^{2}}{27 \sqrt{3}} \approx 1.688$ & 40.8 \\
\hline
\end{tabular}

zero frequency. In practice, the full knowledge of the error kernel is required to make a complete evaluation of the approximation quality. Reducing the sampling density as much as $40 \%$ might keep the behavior for low frequencies similar, but the orthogonal lattice will clearly behave better for high-frequency components as its Nyquist region is much larger. Nevertheless, in the case of equal sampling density, the high value of the area sampling gain indicates a strong advantage for the hexagonal lattice that influences the whole range of the Nyquist region. In practice, the effective gain that can be expected by representing an image on an hexagonal lattice greatly depends on how much its energy is essentially low-pass.

Finally, we put our results in the perspective of the classical observation concerning the approximation of isotropic band-limited signals on hexagonal lattices [9]. For such a signal, an orthogonal lattice needs a density $\frac{2}{\sqrt{3}}$ higher than the one of a hexagonal lattice to satisfy the sampling theorem. This corresponds to $15 \%$ more samples. Our results indicate that the use of ideal reconstruction functions (corresponding to splines with order $L \rightarrow \infty$ ) is not required to exploit the advantage of hexagonal lattices: while a nearest-neighbor scheme $(L=1)$ is clearly too primitive, the second-order hex-spline $(L=2)$ already offers a performance close to the asymptotic behavior $(L \rightarrow \infty)$.

\section{APPENDIX: PROOFS OF EQS. (14) \& (15)}

We first note that $\hat{\eta}_{L}(\boldsymbol{\omega})=\frac{1}{\Omega^{L-1}} \hat{\eta}_{1}(\boldsymbol{\omega})^{L}$. Consequently,

$$
\hat{a}_{\eta_{L}}(\boldsymbol{\omega})=\frac{1}{\Omega^{2 L-2}} \sum_{\mathbf{k} \in \mathbb{Z}^{2}}\left|\hat{\eta}_{1}(\boldsymbol{\omega}-2 \pi \hat{\mathbf{R}} \mathbf{k})\right|^{2 L} .
$$

Hence the expression of the error kernel (see (10)):

$$
\begin{aligned}
E(\boldsymbol{\omega}) & =\frac{\sum_{\mathbf{k} \neq \mathbf{0}}\left|\hat{\eta}_{1}(\boldsymbol{\omega}-2 \pi \hat{\mathbf{R}} \mathbf{k})\right|^{2 L}}{\sum_{\mathbf{k} \in \mathbb{Z}^{2}}\left|\hat{\eta}_{1}(\boldsymbol{\omega}-2 \pi \hat{\mathbf{R}} \mathbf{k})\right|^{2 L}} \\
& =\frac{1}{\Omega^{2 L}} \sum_{\mathbf{k} \neq \mathbf{0}}\left|\hat{\eta_{1}}(\boldsymbol{\omega}-2 \pi \hat{\mathbf{R}} \mathbf{k})\right|^{2 L}+O\left(\|\boldsymbol{\omega}\|^{4 L}\right)
\end{aligned}
$$

because of the conditions in (11) and $\eta_{1}(\mathbf{0})=\Omega$. Therefore

$$
\begin{aligned}
C_{L}^{\text {hex }}(\theta) & =\lim _{\boldsymbol{\omega} \rightarrow \mathbf{0}} \frac{1}{\Omega^{2 L}} \frac{\sum_{\mathbf{k} \neq \mathbf{0}}\left|\hat{\eta}_{1}(\boldsymbol{\omega}-2 \pi \hat{\mathbf{R}} \mathbf{k})\right|^{2 L}}{\|\boldsymbol{\omega}\|^{2 L}} \\
& =\frac{1}{\Omega^{2 L}} \sum_{\mathbf{k} \neq \mathbf{0}}|A(\mathbf{k}, \theta)|^{2 L},
\end{aligned}
$$

where $A(\mathbf{k}, \theta)=\lim _{\boldsymbol{\omega} \rightarrow \mathbf{0}} \hat{\eta_{1}}(\boldsymbol{\omega}-2 \pi \hat{\mathbf{R}} \mathbf{k}) /\|\boldsymbol{\omega}\|$. We obtain the general term in the sum of (14) by calculating the Taylor development of $\eta_{1}(\boldsymbol{\omega})$, whose analytical expression is given in [6]. The terms corresponding to $k 1+k 2 \equiv$ $0[3]$ give the first part of the rhs in (14), if we note that $3 \sum_{n=-\lfloor L / 3\rfloor}^{\lfloor L / 3\rfloor}\left(\begin{array}{l}2 L \\ 3 n+L\end{array}\right)(-1)^{n} \cos (6 n \theta)$ is equal to

$$
2^{2 L}\left[\sin (\theta)^{2 L}+\sin \left(\theta+\frac{2 \pi}{3}\right)^{2 L}+\sin \left(\theta-\frac{2 \pi}{3}\right)^{2 L}\right] .
$$

Hence

$$
C_{L}^{\text {hex* }}=\frac{4 \zeta(2 L)}{\left(24 \sqrt{3} \pi^{2}\right)^{L}}+\left(\frac{27 \sqrt{3}}{32 \pi^{4}}\right)_{k_{2}+k_{1} \neq \equiv 0[3]}^{L}\left[\left(k_{1}+k_{2}\right)\left(k_{1}-2 k_{2}\right)\right]^{-2 L}
$$

After some changes of variables, this last sum turns out to be $2\left(\sum_{n \in \mathbb{Z}}(3 n+1)^{-2 L}\right)^{2}$, which is equal to $2[\zeta(2 L)(1-$ $\left.\left.3^{-2 L}\right)\right]^{2}$. Putting this together yields (15).

\section{REFERENCES}

[1] R. C. Staunton, "The design of hexagonal sampling structures for image digitization and their use with local operators," Image and Vision Computing, vol. 7, no. 3, pp. 162-166, 1989.

[2] M. Unser, "Splines: A perfect fit for signal and image processing," IEEE Signal Proc. Mag., vol. 16, no. 6, Nov. 1999.

[3] T. M. Lehmann, C. Gönner, and K. Spitzer, "Survey: Interpolation methods in medical image processing," IEEE Trans. on Medical Imaging, vol. 18, no. 11, pp. 1049-1075, 1999.

[4] T. Blu, P. Thévenaz, and M. Unser, "Moms: Maximal-order interpolation of minimal support," IEEE Trans. Image Proc., vol. 10, no. 7, pp. 1069-1080, Jul. 2001.

[5] T. Blu and M. Unser, "Quantitative Fourier analysis of approximation techniques: Part I-interpolators and projectors and part II-wavelets," IEEE Trans. Sig. Proc., vol. 47, no. 10, pp. 2783-2806, Oct. 1999.

[6] D. Van De Ville, T. Blu, M. Unser, W. Philips, I. Lemahieu, and R. Van De Walle, "Hex-spline: a novel family for hexagonal lattices," IEEE Trans. Image Proc., vol. 13, no. 6, pp. 758-772, June 2004.

[7] D. Van De Ville, W. Philips, and I. Lemahieu, "Least-squares spline resampling to a hexagonal lattice," Signal Processing: Image Communication, vol. 17, no. 5, pp. 393-408, May 2002.

[8] G. Strang and G. Fix, "A Fourier analysis of the finite element variational method," in Constructive aspect of functional analysis, Rome, Italy: Cremonese, 1971, pp. 796-830.

[9] R. M. Mersereau, "The processing of hexagonally sampled two-dimensional signals," Proc. of the IEEE, vol. 67, no. 6, pp. 930-949, June 1979. 